\title{
The construction of the structural equation model of burden, benefit finding, and anxiety-depression of esophageal cancer caregivers based on Lazarus stress and coping theory
}

\author{
Xiaomeng Wen ${ }^{1,2}$, Danhui Wang ${ }^{1}, \mathrm{Na} \mathrm{Li}^{1}$, Xia Qin ${ }^{1}$, Danfeng Gu${ }^{3}$ \\ ${ }^{1}$ Wuxi School of Medicine, Jiangnan University, Wuxi, China; ${ }^{2}$ Department of Anesthesiology and Perioperative Medicine, The Third Affiliated \\ Hospital of Soochow University, Changzhou, China; ${ }^{3}$ Department of Nursing, Affiliated Hospital of Jiangnan University, Wuxi, China \\ Contributions: (I) Conception and design: X Wen; (II) Administrative support: D Gu; (III) Provision of study materials or patients: X Qin; (IV) \\ Collection and assembly of data: X Wen, D Wang; (V) Data analysis and interpretation: X Wen, N Li; (VI) Manuscript writing: All authors; (VII) \\ Final approval of manuscript: All authors. \\ Correspondence to: Danfeng Gu. Department of Nursing, Affiliated Hospital of Jiangnan University, Wuxi 214062, China. \\ Email: $1308594635 @ q q . c o m$.
}

Backgroundk A large number of studies have shown that the assignment of long-term care duties brings great pressure and negative emotions to caregivers of cancer patients, and also affects the quality of care. Lazarus and Folkman's stress and coping theory holds that the process of cognition and evaluation of stress is key to the stress response when a stressor acts on individuals. This study is to explore the mediating effect of benefit finding between caregiver burden and anxiety-depression of esophageal cancer caregivers, according to a model hypothesis constructed based on stress and coping theory. The design of this study involved correlation and theoretical testing using a structural equation model.

Methods: A total of 228 pairs of esophageal cancer patients from 2 tertiary hospitals and their family caregivers were recruited in this study from May 2020 to January 2021. A questionnaire survey was conducted using the general information questionnaire, the caregiver burden inventory (CBI), the benefit finding scale (BFS), and the hospital anxiety and depression scale (HADS).

Results: A good fitting model [chi-square $\left(\chi^{2}\right) /$ degrees of freedom $(\mathrm{df})=2.212$, root mean square error of approximation $($ RMSEA $)=0.07$, comparative fit index $(\mathrm{CFI})=0.976$, Tucker-Lewis index $(\mathrm{TLI})=0.964$, goodness of fit $(\mathrm{GFI})=0.954$, normed fit index $(\mathrm{NFI})=0.957]$ indicated the mediating effect of benefit finding between caregiver burden and anxiety-depression of esophageal cancer caregivers. A higher level of benefit finding had a negative effect on caregiver burden and anxiety-depression, which reduced the burden and psychological distress of caregivers. The theoretical hypothesis was validated.

Conclusions: Clinical nurses should pay attention to the benefit finding level of caregivers. For caregivers with low-level of benefit finding, intervention measures should be taken to improve the psychological cognitive level of caregivers, which can ultimately improve the quality of life of patients.

Keywords: Esophageal cancer caregivers; benefit finding; caregiver burden; anxiety-depression; mediating effects

Submitted Apr 20, 2021. Accepted for publication Jun 29, 2021.

doi: 10.21037/apm-21-1466

View this article at: https://dx.doi.org/10.21037/apm-21-1466 


\section{Introduction}

The incidence and mortality of esophageal cancer rank the sixth and fourth highest among malignant cancers, respectively (1). The incidence and mortality of esophageal cancer in China account for more than half of those globally (2). Family caregivers take on the main tasks of caring for cancer patients. A large number of studies have shown that the assignment of long-term care duties brings great pressure and negative emotions to caregivers of cancer patients $(3,4)$, and also affects the quality of care $(5,6)$. Lazarus and Folkman's stress and coping theory $(7)$ holds that the process of cognition and evaluation of stress is key to the stress response when a stressor acts on individuals. A successful response produces positive results, otherwise negative results will be produced. Benefit finding (8) is a cognitive behavior evaluation process in which individuals perceive individual, psychological, social, and spiritual benefits after negative events or trauma. The individuals' positive response, reevaluation, optimistic attitude, social support (9-12), intrusive thinking (12), and social constraints have shown a significant correlation with the positive changes of such individuals. Many studies have found (13-15) that benefit finding was the main influencing factor associated with the burden and anxiety-depression of caregivers. Effective improvement of benefit finding can help caregivers adapt to their role of care, increase their positive mood, and their ability to perform as caregivers. At present, there is no study on the mechanism among the 3 factors in China and internationally. Therefore, this study aimed to: (I) construct a structural equation model of benefit finding, caregiver burden, and anxiety-depression based on stress and coping theory, (II) explore the relationship among the benefit finding, caregiver burden, and the anxietydepression of caregivers.

We present the following article in accordance with the SURGE reporting checklist (available at https://dx.doi. org/10.21037/apm-21-1466).

\section{Methods}

\section{Study design}

This study was designed as a cross-sectional study. Esophageal cancer patients from 2 tertiary first-class hospitals in the Jiangsu Province and their main caregivers in their families were selected as participants from May 2020 to January 2021. The inclusion criteria were as follows: (I) patients with pathologically confirmed primary esophageal cancer; (II) the main family caregivers of patients; (III) caregivers with no history of mental disorder and can communicate normally; (IV) caregiving duration $\geq 4$ weeks; (V) patients and their caregivers aged 18 years old or older, and willing to voluntarily participate in the study. The exclusion criteria were as follows: (I) caregivers with an employment relationship to the patient; (II) those who had previously participated in another clinical trial. The sample size was calculated through GPower version 3.1 .9 as follows: validity of sample size was $95.1 \%$, effect size was 0.3 , and significance level was 5\% (bilateral) in 134 respondents. In order to ensure the statistical stability of the structural equation model, the sample size was expanded to $>200$ cases (16). This study adhered to the Helsinki declaration (as revised in 2013), principles of informed consent, security protection, privacy and confidentiality, and fairness and transparency. The study was approved by ethics committees of the 2 hospitals, respectively. The selection and screening process of cases is shown in Figure 1.

All procedures performed in this study involving human participants were in accordance with the Declaration of Helsinki (as revised in 2013). The study was approved by institutional ethics committee of the Third Affiliated Hospital of Soochow University (2020-CL003-01), Affiliated Hospital of Jiangnan University (LS2020010), and informed consent was taken from all the participants.

\section{Research tools}

A general data questionnaire designed by researchers was used to collect patient information (gender, age, disease stages, and payment methods) and that of their caregivers (gender, age, level of education). The modified Chinese version of the benefit finding scale (BFS) (17) was used to measure the level of benefit finding of caregivers. The scale contained 22 items and 5 dimensions: acceptance, family relationship, personal growth, social relations, and health behavior. A 5-point Likert scale method was used [1 point (none) to 5 points (very many)], and the total score range was 22 to 110 points. The higher the score was, the higher the benefit finding level was, and the Cronbach's $\alpha$ coefficient of the scale was 0.93 . The Zarit burden interview (ZBI) (18) was conducted, containing 22 items and 2 dimensions: personal burden and responsibility burden, which used a 4-point Likert scale method [0 points (never) to 4 points (always)], A score of more than 21 points was considered no burden or light burden; 21-39 points: medium burden; and 40 points: severe burden. The 


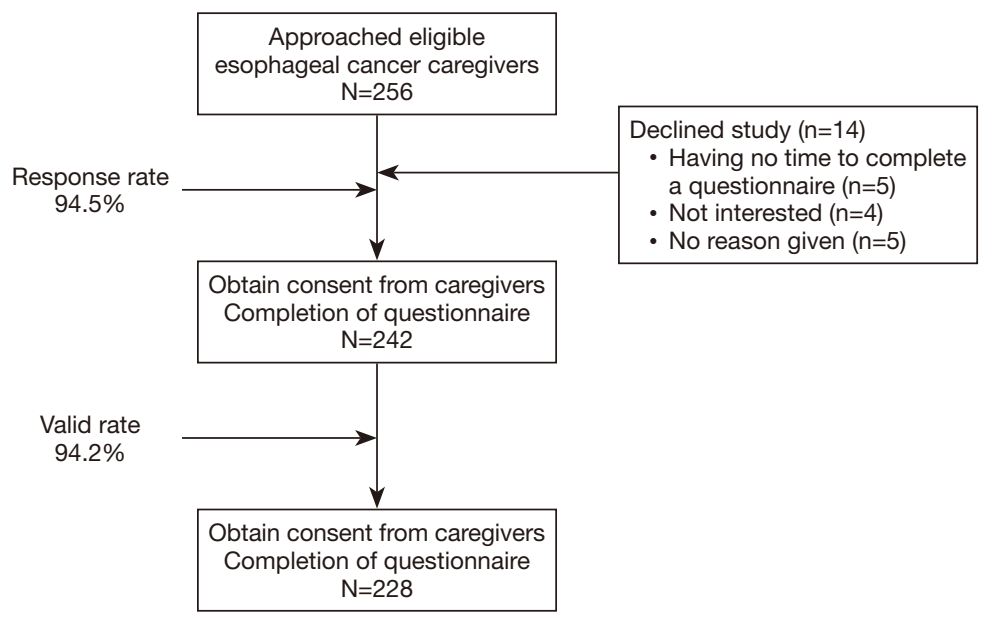

Figure 1 The study flowchart.

Cronbach's $\alpha$ coefficient of ZBI in the Chinese version was 0.86 (19). The hospital anxiety and depression scale (HADS) was used as a reliable tool for screening emotional disorders (20); it included an anxiety and depression subscale, which incorporated 14 items. Each item adopted a 4-point Likert scale (0-3 points), whereby a higher score indicated more serious emotions of anxiety and depression, and the study used 9-point as the cut-off value (21) to judge the anxiety and depression of caregivers.

\section{Reliability and effectiveness}

A preliminary study involving 20 respondents was conducted to evaluate reliability and management issues. The results showed that the Cronbach's $\alpha$ coefficient of the questionnaire was acceptable. The Cronbach's $\alpha$ coefficient of BFS in previous studies was 0.93 (17), that of caregiver burden inventory (CBI) was $0.86(19)$, and those of anxiety and depression subscale were $0.68-0.93$ (average 0.83 ) and $0.67-0.90$ (average 0.82 ), respectively $(22,23)$. The results of these previous studies confirmed the effectiveness and reliability of the research tools designated to this study.

\section{Data collection}

The questionnaire survey method was adopted for data collection. Before the interview, informed consent was provided by all participants, entailing the study purpose, the voluntary nature of participation in the study, and the confidentiality of privacy and anonymous processing of information. All participants completed the questionnaire within $20 \mathrm{~min}$. The researchers checked the completed questionnaire to evaluate validity of the responses to the questionnaire and eliminate any questionnaires with invalid answers.

\section{Statistical analysis}

The software SPSS25.0 (IBM Corp., Armonk, NY, USA) and AMOS 25.0 (IBM Corp.) were used for statistical analysis. For descriptive data, average, standard deviation (SD), frequency, and percentage were used to describe and summarize the demographic characteristics of the study, and the Kolmogorov-Smirnov test was used to test the normal distribution of data. If data (including BFS, CBI, and HADS) did not conform to normal distribution, the median and quartile spacing were used. Spearman's correlation was used to describe the correlation between BFS, CBI, and HADS. The correlation coefficients ( $r)<0.25,0.25-0.50$, $0.50-0.75$, and $>0.75$ indicated general, medium, good, and excellent, respectively (24).

In order to explore the effect of benefit finding on caregiver burden and anxiety-depression of caregivers, we constructed a structural equation model based on stress and coping theory. Benefit finding was used as the intermediary variable of caregiver burden and anxiety-depression of caregivers. The relationship between the study variables was determined by using maximum likelihood estimation and path analysis. The bootstrap deviation correction method was used to test the $95 \%$ confidence interval (CI) for the nonstandard coefficient in order to evaluate the significance of the total effect and the total relationship. 


\section{Results}

\section{General information of patients with esophageal cancer and their caregivers}

There were 228 pairs of patients and their main caregivers in this study. The caregivers were $20-85$ years old, with an average age of $(60.86 \pm 7.44)$ years; 43 males $(18.9 \%)$ and 185 females $(81.1 \%)$; the care time was $1-234$ months. The patients were $42-87$ years old, with an average age of $(67.20 \pm 7.44)$ years old, 198 males $(86.8 \%)$ and 30 females (13.2\%). There were 7 patients in tumor, node, metastasis (TNM) stage I (3.1\%), 74 in TNM stage II (32.5\%), 85 in stage III $(37.3 \%)$, and 62 in stage IV (27.2\%). Other general information of patients and caregivers is shown in Table 1.

\section{The scores of BFS, CBI, and HADS}

The total score of BFS was 80 [51-90], and the 5 dimensions were: acceptance, 11 [7-13]; family relationship, 21 [14-24]; personal growth, 27 [19-31]; social relationship, 10 [7-12]; and health behavior, 9 [7-12], respectively. The total score of CBI was 34 [23-41], and the 2 dimensions were: personal burden, 18 [14-24]; and responsibility burden, 8 [5-11]. There were $26(11.4 \%)$ patients with no or light burden, 128 (56.1\%) with medium burden, and $74(32.5 \%)$ with heavy burden. The total score of HADS was 18 [10-26], among which anxiety was 9 [5-12], and depression was 8 [5-13]. There were 116 (50.9\%) patients with anxiety, 106 (46.5\%) with depression, and 107 (46.9\%) with both anxiety and depression.

\section{Correlation analysis of benefit finding, caregiver burden, and anxiety-depression of caregivers}

As shown in Table 2, caregiver burden was negatively correlated with benefit finding. The score of each dimension was positively correlated with anxiety-depression, and negatively correlated with benefit finding.

\section{Mediating effect analysis}

Correlation analysis showed that there was a significant correlation among benefit finding, caregiver burden, and anxiety-depression of caregivers. The structural equation model was constructed according to the stress and coping theory, and caregiver burden was taken as the stress source of caregivers. The outcome variable was anxiety-depression, and the mediating variable was benefit finding. As shown in Figure 2. The fitting results of the model: $\chi^{2}=53.09$, degrees of freedom $(\mathrm{df})=24, \mathrm{P}=0.001, \chi^{2} / \mathrm{df}=2.212$, root mean square error of approximation (RMSEA) $=0.073$, comparative fit index $(\mathrm{CFI})=0.976$, Tucker-Lewis index $(\mathrm{TLI})=0.964$, goodness of fit $(\mathrm{GFI})=0.954$, normed fit index (NFI), which indicated that the fitting degree of the model was good. The path from caregiver burden to anxietydepression $(\gamma=0.42, \mathrm{SE}=0.124, \mathrm{P}<0.001)$, the path from caregiver burden to benefit finding $(\gamma=-0.53, \mathrm{SE}=0.098$, $\mathrm{P}<0.001)$, and the path from benefit finding to anxietydepression $(\gamma=-0.63, \mathrm{SE}=0.126, \mathrm{P}<0.001)$. Bootstrap bias correction method was used to test the mediating effect of the model. The results showed that the path coefficient of caregiver burden $v s$. anxiety-depression was 0.40 (95\% CI: 0.174 to 0.721 ), and the path coefficient of benefit finding between caregiver burden and anxiety-depression was 0.32 (95\% CI: 0.179 to 0.582 ). The CI did not include 0 . The mediating effect and hypothesis were validated.

\section{Discussion}

\section{The effect of caregiver burden on anxiety-depression in esophageal cancer caregivers}

In this study, there were $202(88.6 \%)$ esophageal cancer caregivers with medium to severe burden, which was slightly higher than the report of $\mathrm{Hu}$ et al. (25) on the caregivers of lung cancer patients. This may have been due to the fact that the majority of patients with esophageal cancer were elderly patients, most of them were in the middle and late stage due to their disease characteristics (26). In this study, the caregivers were older, with $63.2 \%(\mathrm{n}=144)$ over 60 years old. In the process of care, the caregivers were prone to lack of strength and fatigue, which led to them experiencing an increased burden. In the process of caring for patients with malignant tumors, the main caregivers of the family usually took on heavy care tasks (27). The longterm care, economic burden caused by repeated treatment, caregiver worry about the disease and prognosis (28), and weakening of social relations led to anxiety-depression. However, the study also found that caregivers who bore the same burden of care did not necessarily experience the same level of anxiety and depression, which indicated that the caregiver burden was restricted by other factors. The results of analysis showed that benefit finding was negatively correlated with the burden and anxiety-depression of caregivers, indicating that the benefit finding of caregivers had an impact on their burden and anxiety-depression. 
Table 1 General information of 228 patients with esophageal cancer and their caregivers

\begin{tabular}{|c|c|}
\hline Item & n (\%) \\
\hline \multicolumn{2}{|l|}{ Caregivers } \\
\hline \multicolumn{2}{|l|}{ Gender } \\
\hline Male & $43(18.9)$ \\
\hline Female & $185(81.1)$ \\
\hline \multicolumn{2}{|l|}{ Age (year) } \\
\hline$<45$ & $19(8.3)$ \\
\hline $45-59$ & $65(28.5)$ \\
\hline$\geq 60$ & $144(63.2)$ \\
\hline \multicolumn{2}{|l|}{ Current occupational status } \\
\hline Yes & $64(28.1)$ \\
\hline No & $111(48.7)$ \\
\hline Retired & $53(23.2)$ \\
\hline \multicolumn{2}{|l|}{ Residential area } \\
\hline Urban & $78(34.2)$ \\
\hline Town & $150(65.8)$ \\
\hline \multicolumn{2}{|l|}{ Relationship between caregivers and patients } \\
\hline Spouse & $183(80.3)$ \\
\hline Children & $40(17.5)$ \\
\hline Parent & $1(0.4)$ \\
\hline Other & $4(1.8)$ \\
\hline \multicolumn{2}{|l|}{ Daily care time (hours) } \\
\hline$<6$ & $25(11.0)$ \\
\hline $6-12$ & $106(46.5)$ \\
\hline$>12$ & $97(42.5)$ \\
\hline \multicolumn{2}{|l|}{ Per capita monthly income of family (yuan) } \\
\hline $1,000-3,000$ & $123(53.9)$ \\
\hline $3,001-5,000$ & $46(20.2)$ \\
\hline $5,001-8,000$ & $40(17.5)$ \\
\hline $8,001-10,000$ & $13(5.7)$ \\
\hline$>10,000$ & $6(2.6)$ \\
\hline \multicolumn{2}{|l|}{ Education } \\
\hline Primary and junior high schools & $174(76.3)$ \\
\hline High school/technical secondary school & $23(10.1)$ \\
\hline Junior college or above & $31(13.6)$ \\
\hline
\end{tabular}

Table 1 (continued)
Table 1 (continued)

\begin{tabular}{|c|c|}
\hline Item & $\mathrm{n}(\%)$ \\
\hline \multicolumn{2}{|l|}{ Duration of caregiving role (month) } \\
\hline$<6$ & $114(50)$ \\
\hline $6-11$ & $35(15.4)$ \\
\hline$\geq 12$ & $79(34.6)$ \\
\hline \multicolumn{2}{|l|}{ Chronic disease } \\
\hline No & $118(51.8)$ \\
\hline 1 & $80(35.1)$ \\
\hline 2 or more & $30(13.2)$ \\
\hline \multicolumn{2}{|l|}{ Other } \\
\hline \multicolumn{2}{|l|}{ Caregivers number } \\
\hline No & $136(59.6)$ \\
\hline 1 & $72(31.6)$ \\
\hline 2 or more & $20(8.8)$ \\
\hline \multicolumn{2}{|l|}{ Patients } \\
\hline \multicolumn{2}{|l|}{ Gender } \\
\hline Male & $198(86.8)$ \\
\hline Female & $30(13.2)$ \\
\hline \multicolumn{2}{|l|}{ Age (year) } \\
\hline$<45$ & $1(0.4)$ \\
\hline $45-59$ & $36(15.8)$ \\
\hline$\geq 60$ & $191(83.8)$ \\
\hline \multicolumn{2}{|l|}{ Payment method } \\
\hline Employee medical insurance & $77(33.8)$ \\
\hline Resident medical insurance & $41(18.0)$ \\
\hline Commercial insurance & $6(2.6)$ \\
\hline New rural cooperative medical insurance & $103(45.2)$ \\
\hline Own expense & $1(0.4)$ \\
\hline \multicolumn{2}{|l|}{ Disease staging } \\
\hline I & $7(3.1)$ \\
\hline ॥ & $74(32.5)$ \\
\hline III & 85 (37.3) \\
\hline IV & $62(27.2)$ \\
\hline \multicolumn{2}{|l|}{ Treatment } \\
\hline Chemotherapy & $163(71.5)$ \\
\hline Radiotherapy & $23(10.1)$ \\
\hline Chemotherapy + radiotherapy & $26(11.4)$ \\
\hline Other & $16(7.0)$ \\
\hline
\end{tabular}




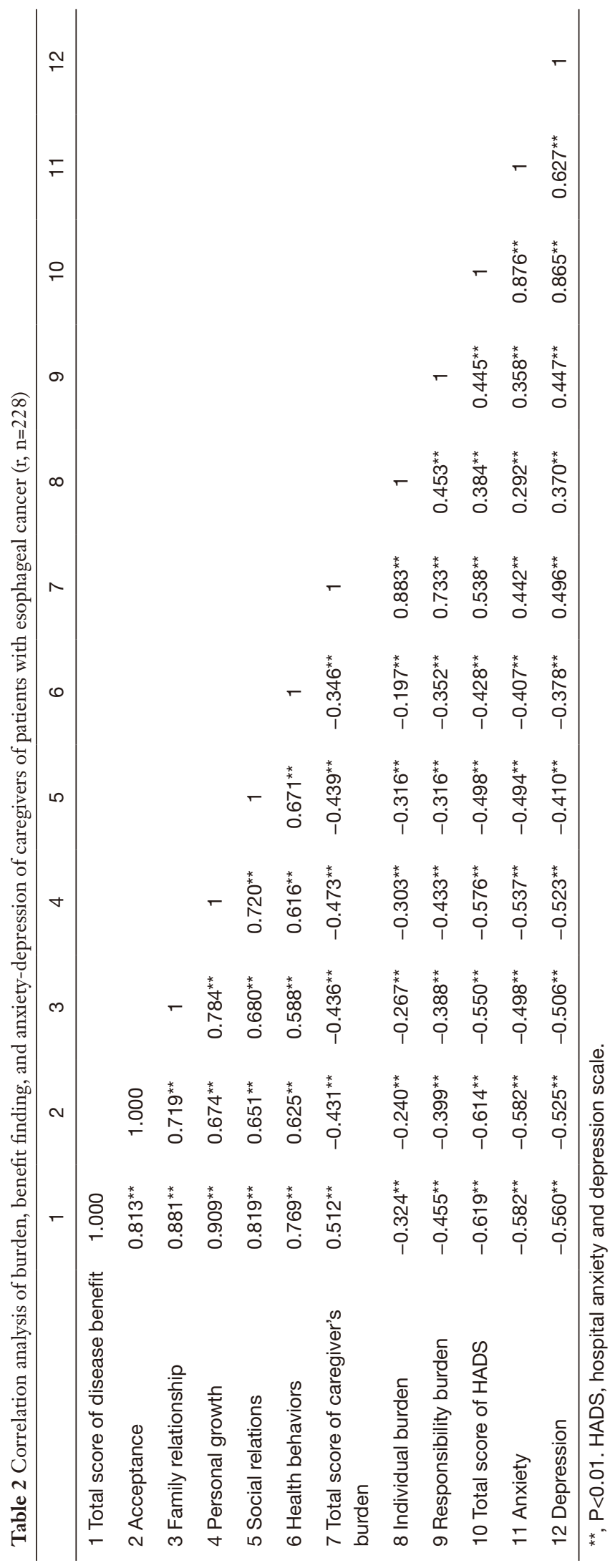

\section{The effect of caregiver burden on anxiety-depression in caregivers of esophageal cancer patients}

Fletcher et al.'s experience model of cancer family care (29) showed that the diagnosis and treatment of cancer can initiate the stress response of patients and their families. When stress acted on individuals, the individual response measures and cognitive evaluation behaviors engendered psychological and physical health results, and this cognitive evaluation behavior can be used in the whole treatment. Previous studies $(30,31)$ have shown that the caregivers of cancer patients who had a higher level of benefit finding, had a lower level of anxietydepression. Through positive cognitive evaluation behavior, the benefits and growth of caregivers were important factors in their response to stressors. In this survey, the majority of caregivers of esophageal cancer patients were spouses of the patient (183/80.3\%), and as the continual close companion of the patients, they played an important role in patient recovery (32). When the patients and their spouses were a team coping with stress events, stress perception was mutually influenced (33). A positive response of both spouses helped reduce the load of stress and negative events on individuals. However, caregivers often neglected their own health status because of heavy care tasks; in particular, spouses of elderly patients, who were aged similarly to the patients and had their own chronic diseases, were more likely to experience anxiety and depression (34). Medical staff should approach patients and their spouses as a unified dyad (35), as doing so can help them effectively identify adverse stress reactions, improve their methods of stress cognition, and enhance the caregiver's ability to implement caregiver adaptation and active response.

\section{The mediating effect of benefit finding on caregiver burden and anxiety-depression of caregivers}

The results of path analysis of the structural equation model showed that the caregiver's benefit finding contributed a mediating role in the caregiver burden and anxietydepression of caregivers. This results supported Larzarus' stress and coping theory (7). The theory holds that individuals can initiate self-reliance when responding to stress. Both internal and external response resources should be drawn on to maintain the balance of the internal and external environments, including cognitive evaluation of stress, so as to alleviate and adjust their stress response, produce positive response measures, and maintain the individual's mental and physical health. Previous studies 


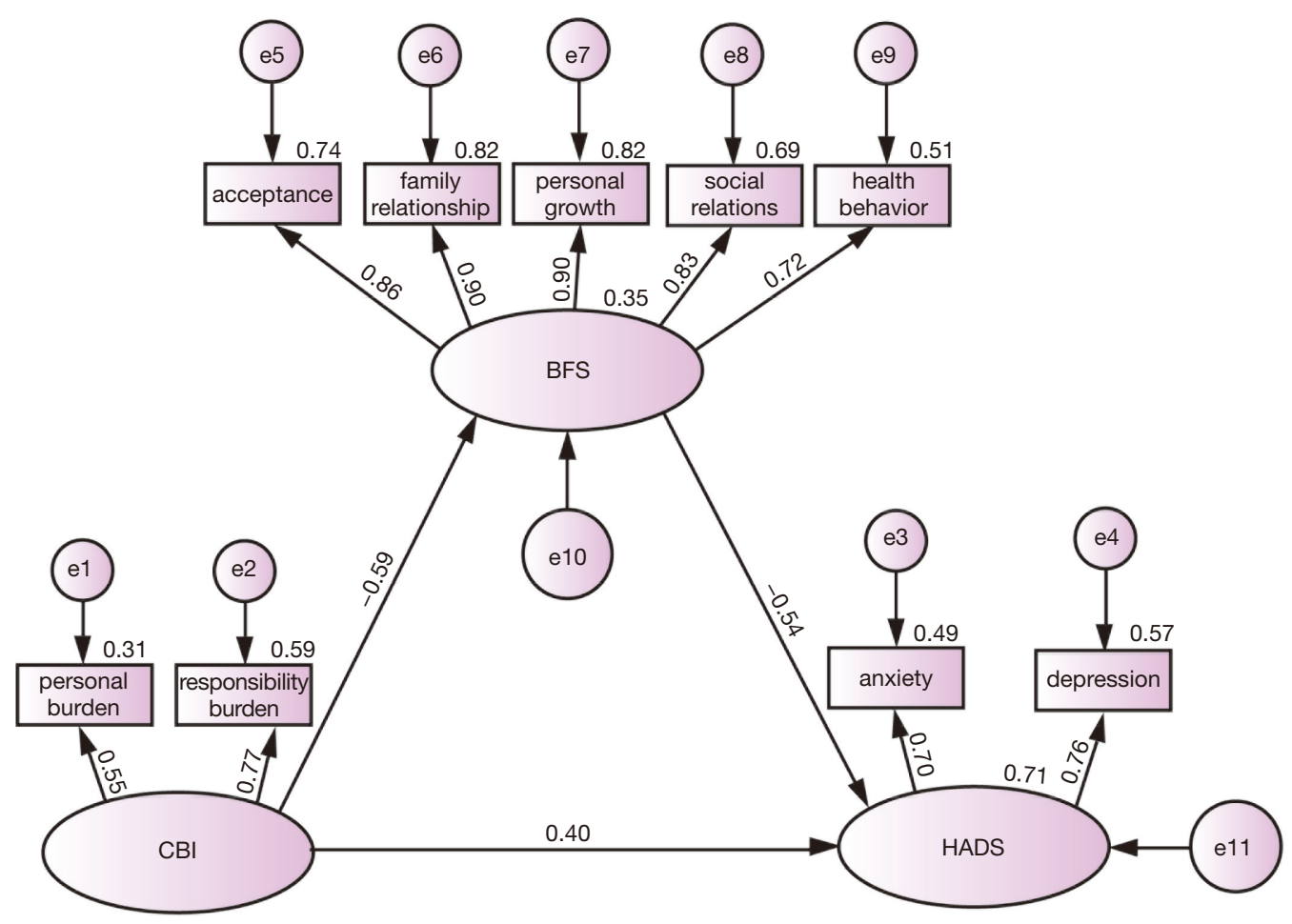

Figure 2 Structural equation model of caregivers' benefit finding. BFS, benefit finding scale; CBI, caregiver burden inventory; HADS, hospital anxiety and depression scale.

$(30,36)$ have shown that, as an individual cognitive evaluation strategy, benefit finding played an active role in the process of response to diseases among family caregivers of cancer patients, such as orderly arrangement of family life, positive self-discovery, and enhancement of family relations (37). Positive cognitive state can affect the individual's mental state and behavior, help them face difficulties calmly, reduce their bad psychological state, improve the caregiver's adaptation to the role of care (38), and thus improve the patient's disease treatment and rehabilitation. Therefore, the results of this study suggested that caregivers should be targeted to improve their psychological cognitive level and positive response strategies. At present, international interventions on benefit finding mainly involved cognitive behavioral therapy (39), brief writing intervention (40), and web-based meaningcentered psychotherapy (41). At present, the benefit finding intervention of Chinese control patients is still in the initial stage. In the future research, we should pay attention to the intervention of benefit finding, so as to reduce the caregiver's anxiety-depression, improve the quality of care for patients.

\section{The limitations of this study}

There were some limitations to this study. Firstly, the study implemented a cross-sectional design, only the independent time point data were collected, and the data of caregivers in different treatment and care stages could not be evaluated. In the later stage, a longitudinal research design could be introduced to show the trend of caregiver benefit finding with the course of time. Secondly, the factors associated with psychological problems of caregivers included in this study were limited; later research was conducted, and it was suggested that the relevant factors of anxiety-depression should be discussed comprehensively based on the theoretical framework, which can provide the basis for effective intervention measures.

\section{Acknowledgments}

Funding: This study was funded by the Wuxi grant (MS201945). 


\section{Footnote}

Reporting Checklist: The authors have completed the SURGE reporting checklist. Available at https://dx.doi. org/10.21037/apm-21-1466

Data Sharing Statement: Available at https://dx.doi. org/10.21037/apm-21-1466

Conflicts of Interest: All authors have completed the ICMJE uniform disclosure form (available at https://dx.doi. org/10.21037/apm-21-1466). The authors have no conflicts of interest to declare.

Ethical Statement: The authors are accountable for all aspects of the work in ensuring that questions related to the accuracy or integrity of any part of the work are appropriately investigated and resolved. All procedures performed in this study involving human participants were in accordance with the Declaration of Helsinki (as revised in 2013). The study was approved by institutional ethics committee of the Third Affiliated Hospital of Soochow University (2020-CL003-01), Affiliated Hospital of Jiangnan University (LS2020010), and informed consent was taken from all the participants.

Open Access Statement: This is an Open Access article distributed in accordance with the Creative Commons Attribution-NonCommercial-NoDerivs 4.0 International License (CC BY-NC-ND 4.0), which permits the noncommercial replication and distribution of the article with the strict proviso that no changes or edits are made and the original work is properly cited (including links to both the formal publication through the relevant DOI and the license). See: https://creativecommons.org/licenses/by-nc-nd/4.0/.

\section{References}

1. Mao YS, Gao SG, Wang Q, et al. Epidemiological characteristic and current status of surgical treatment for esophageal cancer by analysis of national registry database. Zhonghua Zhong Liu Za Zhi 2020;42:228-33.

2. Shang QX, Yang YS, Hu WP, et al. Clinical and prognostic significance of preoperative lymphocyte-monocyte ratio, neutrophil-lymphocyte ratio and neutrophil-monocyte ratio on esophageal squamous cell carcinoma patients. Transl Cancer Res 2020;9:3903-14.

3. Sklenarova H, Krümpelmann A, Haun MW, et al. When do we need to care about the caregiver? Supportive care needs, anxiety, and depression among informal caregivers of patients with cancer and cancer survivors. Cancer 2015;121:1513-9.

4. Shin JY, Lim JW, Shin DW, et al. Underestimated caregiver burden by cancer patients and its association with quality of life, depression and anxiety among caregivers. Eur J Cancer Care (Engl) 2018;27:e12814.

5. Nipp RD, El-Jawahri A, Fishbein JN, et al. Factors associated with depression and anxiety symptoms in family caregivers of patients with incurable cancer. Ann Oncol 2016;27:1607-12.

6. Johansen S, Cvancarova M, Ruland C. The Effect of Cancer Patients' and Their Family Caregivers' Physical and Emotional Symptoms on Caregiver Burden. Cancer Nurs 2018;41:91-9.

7. Folkman S, Lazarus RS, Pimley S, et al. Age differences in stress and coping processes. Psychol Aging 1987;2:171-84.

8. Pascoe L, Edvardsson D. Benefit finding in cancer: a review of influencing factors and health outcomes. Eur J Oncol Nurs 2013;17:760-6.

9. Helgeson VS, Reynolds KA, Tomich PL. A meta-analytic review of benefit finding and growth. J Consult Clin Psychol 2006;74:797-816.

10. Sears SR, Stanton AL, Danoff-Burg S. The yellow brick road and the emerald city: benefit finding, positive reappraisal coping and posttraumatic growth in women with early-stage breast cancer. Health Psychol 2003;22:487-97.

11. Schulz U, Mohamed NE. Turning the tide: benefit finding after cancer surgery. Soc Sci Med 2004;59:653-62.

12. Dunn J, Occhipinti S, Campbell A, et al. Benefit finding after cancer: the role of optimism, intrusive thinking and social environment. J Health Psychol 2011;16:169-77.

13. Gardner MH, Mrug S, Schwebel DC, et al. Demographic, medical, and psychosocial predictors of benefit finding among caregivers of childhood cancer survivors. Psychooncology 2017;26:125-32.

14. Lum HD, Lo D, Hooker S, et al. Caregiving in heart failure: relationship quality is associated with caregiver benefit finding and caregiver burden. Heart Lung 2014;43:306-10.

15. Cassidy T. Benefit finding through caring: the cancer caregiver experience. Psychol Health 2013;28:250-66.

16. Torres GCS, Relf MV, Tuazon JA. The mediating role of pre-operative patient readiness on surgical outcomes: A structural equation model analysis. J Adv Nurs 2020;76:1371-83. 
17. Bian J, Zhang LF, Liu ZZ, et al. Reliability and Validity of the Revised Chinese Version of Benefit Finding Scale in Family Caregivers of Cancer Patients. Chinese General Practice, 2018. doi:10.3969/j.issn.1007-9572.2018.00.222.

18. Zarit SH, Orr NK, Zarit JM. The Hidden Victims of Alzheimer's Disease: Families Under Stress; 1985.

19. Sun X, Ge J, Meng H, et al. The Influence of Social Support and Care Burden on Depression among Caregivers of Patients with Severe Mental Illness in Rural Areas of Sichuan, China. Int J Environ Res Public Health 2019;16:1961.

20. Zigmond AS, Snaith RP. The hospital anxiety and depression scale. Acta Psychiatr Scand 1983;67:361-70.

21. Olssøn I, Mykletun A, Dahl AA. The Hospital Anxiety and Depression Rating Scale: a cross-sectional study of psychometrics and case finding abilities in general practice. BMC Psychiatry 2005;5:46.

22. Li Q, Lin Y, Zhou H, et al. Factors moderating the mutual impact of benefit finding between Chinese patients with cancer and their family caregivers: A cross-sectional study. Psychooncology 2018;27:2363-73.

23. Lee YH, Liao YC, Liao WY, et al. Anxiety, depression and related factors in family caregivers of newly diagnosed lung cancer patients before first treatment. Psychooncology 2013;22:2617-23.

24. Fortney LG, Watkins MP. Foundations of Clinical Research: Applications to Practice. Connecticut Appleton \& Lange, 2013:53.

25. Hu X, Peng X, Su Y, et al. Caregiver burden among Chinese family caregivers of patients with lung cancer: A cross-sectional survey. Eur J Oncol Nurs 2018;37:74-80.

26. Chen R, Zheng RS, Zhang SW, et al. Analysis of incidence and mortality of esophageal cancer in China, 2015. Zhonghua Yu Fang Yi Xue Za Zhi 2019;53:1094-7.

27. Zhu XR, Zhao T, Gu H, et al. High risk of anxiety and depression in caregivers of adult patients with epilepsy and its negative impact on patients' quality of life. Epilepsy Behav 2019;90:132-6.

28. Tao X, Chow SKY, Zhang H, et al. Family caregiver's burden and the social support for older patients undergoing peritoneal dialysis. J Ren Care 2020;46:222-32.

29. Fletcher BS, Miaskowski C, Given B, et al. The cancer family caregiving experience: an updated and expanded conceptual model. Eur J Oncol Nurs 2012;16:387-98.

30. Lin Y, Luo X, Li J, et al. The dyadic relationship of benefit finding and its impact on quality of life in colorectal cancer survivor and spousal caregiver couples. Support Care Cancer 2021;29:1477-86.
31. Li Q, Chiang VC, Xu X, et al. The Experiences of Chinese Couples Living With Cancer: A Focus Group Study. Cancer Nurs 2015;38:383-94.

32. Hsu T, Nathwani N, Loscalzo M, et al. Understanding Caregiver Quality of Life in Caregivers of Hospitalized Older Adults With Cancer. J Am Geriatr Soc 2019;67:978-86.

33. Li Q, Xu Y, Zhou H, et al. Factors influencing the healthrelated quality of life of Chinese advanced cancer patients and their spousal caregivers: a cross-sectional study. BMC Palliat Care 2016;15:72.

34. Adelman RD, Tmanova LL, Delgado D, et al. Caregiver burden: a clinical review. JAMA 2014;311:1052-60.

35. Schulz R. The Future of Caregiver Efficacy Research: Commentary on "Long-Term Outcomes of the BenefitFinding Group Intervention for Alzheimer Family Caregivers". Am J Geriatr Psychiatry 2019;27:995-7.

36. Rodrigue JR, Dimitri N, Reed A, et al. Spouse caregivers of kidney transplant patients: quality of life and psychosocial outcomes. Prog Transplant 2010;20:335-42; quiz 343.

37. Jansen L, Hoffmeister M, Chang-Claude J, et al. Benefit finding and post-traumatic growth in long-term colorectal cancer survivors: prevalence, determinants, and associations with quality of life. Br J Cancer 2011;105:1158-65.

38. McLennon SM, Habermann B, Rice M. Finding meaning as a mediator of burden on the health of caregivers of spouses with dementia. Aging Ment Health 2011;15:522-30.

39. Antoni MH, Lehman JM, Kilbourn KM, et al. Cognitivebehavioral stress management intervention decreases the prevalence of depression and enhances benefit finding among women under treatment for early-stage breast cancer. Health Psychol 2001;20:20-32.

40. Brand C, O'Connell BH, Gallagher S. A randomised controlled trial of benefit finding in caregivers: The Building Resources in Caregivers Study Protocol. Health Psychol Open 2015;2:2055102915595019.

41. Applebaum AJ, Buda KL, Schofield E, et al. Exploring the cancer caregiver's journey through web-based MeaningCentered Psychotherapy. Psychooncology 2018;27:847-56.

(English Language Editor: J. Jones)

Cite this article as: Wen X, Wang D, Li N, Qin X, Gu D. The construction of the structural equation model of burden, benefit finding, and anxiety-depression of esophageal cancer caregivers based on Lazarus stress and coping theory. Ann Palliat Med 2021;10(7):7644-7652. doi: 10.21037/apm-21-1466 